\title{
A case of cavernous carotid aneurysm diagnosed when diplopia developed after endoscopic sinus surgery*
}

\author{
Masayoshi Tei ${ }^{1,2}$, Eri Mori², Hiromi Kojima², Nobuyoshi Otori ${ }^{2}$ \\ 'SUBARU Health Insurance Society Ota Memorial Hospital, Department of Otorhinolaryngology, Oshimacho, Ota, \\ Gunma, Japan \\ 2The Jikei University School of Medicine, Otorhinolaryngology-Head and Neck Surgery, Nishi-Shimbashi, \\ Minato-ku, Tokyo, Japan
}

Rhinology Online, Vol 3: 178 - 183, 2020

http://doi.org/10.4193/RHINOL/20.076

*Received for publication:

October 11,2020

Accepted: October 26, 2020

Published: November 14, 2020

\begin{abstract}
Background: Visual complications of endoscopic sinus surgery usually occur during or immediately after the surgery. We report a case of cavernous carotid aneurysm which developed and gradually worsened after endoscopic sinus surgery was performed.

Case presentation: A 63-year-old woman with chronic rhinosinusitis resistant to conservative treatment underwent endoscopic sinus surgery. Despite the surgery being successful and without complications, diplopia developed 2 weeks later. Intracranial imaging revealed a giant cavernous carotid aneurysm as a likely cause of the diplopia. The patient underwent endovascular stenting treatment, and the diplopia was consequently reduced.
\end{abstract}

Conclusions: We experienced a rare case of cavernous carotid aneurysm which started to develop 2 weeks after endoscopic sinus surgery. Possible causes of the aneurysm in this patient are an indirect effect of surgery, such as perioperative hypertension, and bacterial sinusitis.

Key words: craniocerebral trauma, nasal surgical procedures, sinusitis, sphenoid sinus

\section{Introduction}

Nasal and paranasal diseases can have orbital complications and cause visual impairment owing to their adjacent locations. Surgical treatment for paranasal sinuses can also have visual complications, which rarely occur long after surgery. We report a case of cavernous carotid aneurysm (CCA) diagnosed when diplopia developed and gradually worsened after endoscopic sinus surgery (ESS) was performed.

\section{Case presentation}

A 63-year-old woman with no known preexisting illnesses underwent ESS for chronic rhinosinusitis resistant to conservative treatment. Preoperative computed tomography (CT) showed bilateral nasal polyps and opacification in the sinuses (Figure 1). With a biopsy of the nasal polyps, eosinophilic chronic rhinosinusitis was diagnosed. This condition was treated by the department of otorhinolaryngology by means of ESS, septoplasty, and submucosal inferior conchotomy with polypectomy and total removal of sinus laminae. The surgery lasted 2 hours 9 minutes under general anesthesia with desflurane for 2 hours 57 minutes. Vital signs during the surgery were stable; blood pressure was maintained at 80 to 100 over 50 to $70 \mathrm{~mm} \mathrm{Hg}$ with a total blood loss of $60 \mathrm{ml}$. No intraoperative trauma occurred, and postoperative endoscopic examinations showed sinus membranes without swelling or infections. Postoperative medications include oral macrolide, carbocisteine, antihistamine, prednisolone $2.5 \mathrm{mg}$ and topical steroid.

Two weeks after ESS the patient noticed slight diplopia with far vision. Diplopia due to esotropia was diagnosed 5 weeks after ESS at a nearby private ophthalmologic clinic and was treated with prism correction. The ophthalmologic clinic reported esotropia of $2^{\Delta}$ (prism dioptres) during the first visit, $10^{\Delta} 7$ weeks later ( 12 weeks after ESS), and $18^{\Delta} 14$ weeks later ( 19 weeks after ESS). The patient had reported the diplopia to our department 7 weeks after ESS; however, we assumed the diplopia was not rela- 


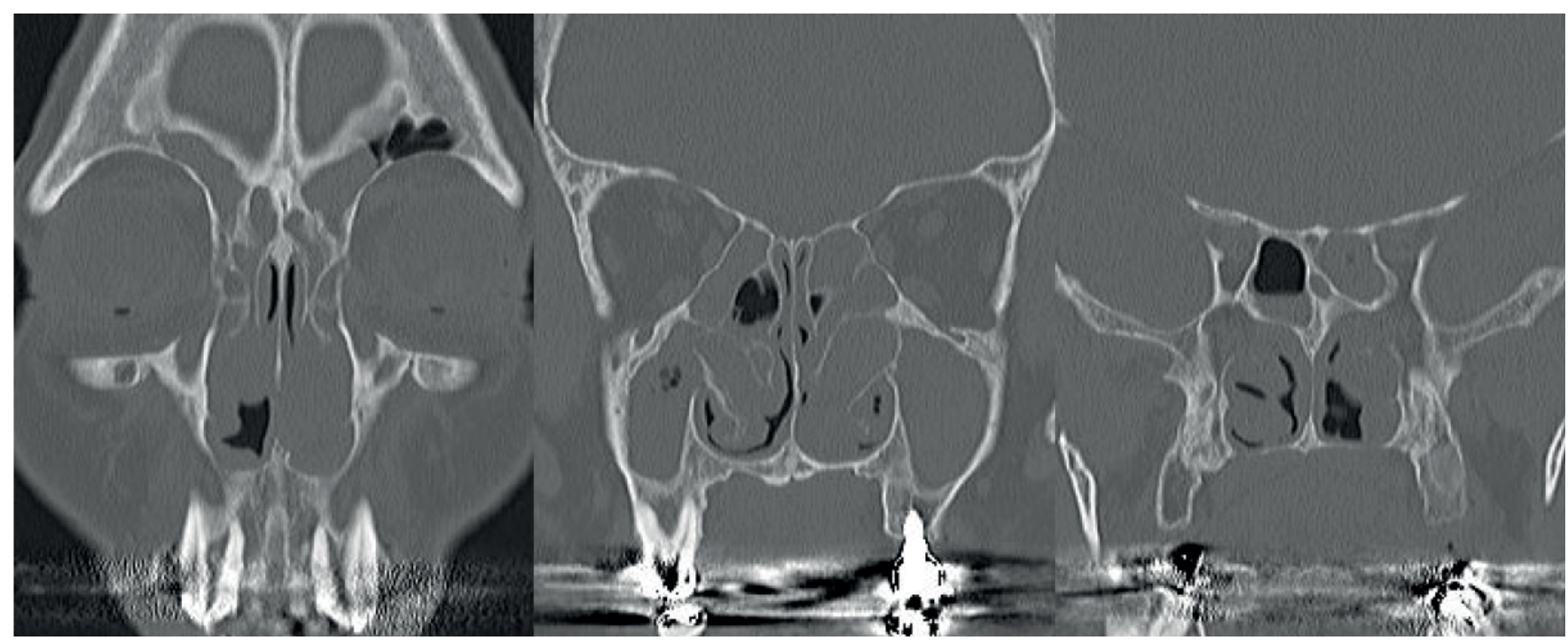

Figure 1. Preoperative sinus computed tomography. Bilateral opacification of frontal, ethmoid, maxillary sinuses, and nasal polyps. Findings of computed tomography strongly indicate eosinophilic chronic rhinosinusitis.

ted to ESS because it had started 2 weeks after ESS and because endoscopic findings of the sinus membranes remained intact postoperatively. She did not report any changes of the diplopia during the following 2 visits to our department ( 9 and 13 weeks after ESS) but complained of worsening in a later visit (20 weeks after ESS). Therefore, we referred the patient to our hospital's department of neurology to investigate possible intracranial causes.

Magnetic resonance imaging/angiography performed 22 weeks after ESS at our hospital's department of neurology showed multiple intracranial aneurysms. A detailed $C T$ angiographic examination (Figure $2 A, B, C$ ) showed right $C C A$, which was the largest aneurysm, with a maximum diameter of $17 \mathrm{~mm}$, and was believed to be causing diplopia. A Hess screen test performed 26 weeks after ESS at our hospital's department of ophthalmology suggested esotropia of the right eye (Figure $2 \mathrm{D}$ ). Ten weeks the diagnosis of aneurysm (32 weeks after ESS) the patient was admitted to another hospital, where she underwent endovascular aneurysm stenting treatment. After this treatment the diplopia decreased owing to improved eye movement (Figure $2 \mathrm{D}$ ). The time course of esotropia and its related events is also shown (Figure $2 \mathrm{E}$ ). The patient did not complain of any nasal symptoms in subsequent visits to our department, and no signs or endoscopic findings suggested the recurrence of sinusitis.

\section{Discussion}

Visual complications of ESS often arise during or immediately after surgery and are usually due to intraoperative trauma. A meta-analysis in 1994 found that the rate of visual complications after endoscopic surgery was $0.12 \%{ }^{(1)}$ and was lower than after traditional surgery $(0.47 \%)$. Studies in the 2010 s have found rates of orbital injury after ESS of $0.07 \%{ }^{(2)}$ and $0.09 \%{ }^{(3)}$. Most of these complications were orbital hematomas with only a few cases of extraocular muscle injury (2 of 57 cases of orbital injury) requiring reconstructive surgery ${ }^{(3)}$. In our patient, intraoperative trauma did not occur, and surgery-related complications typically would have appeared within 2 weeks.

Of cerebral aneurysms, fewer than $1 \%$ are traumatic intracranial aneurysms ${ }^{(4)}$. Intracranial carotid aneurysm due to artery injury has been reported after endoscopic endonasal skull base surgery but rarely after ESS. However, at least 4 cases of intracranial carotid aneurysm after ESS have been reported: 1 in the ethmoidal sinus after skull base injury ${ }^{(5)}, 1$ in the sphenoid sinus due to lateral wall injury ${ }^{(6)}$, and 2 with subarachnoid hemorrhage after functional ESS and cosmetic rhinoplasty ${ }^{(7)}$. latrogenic traumatic aneurysms are most often caused by direct arterial injury, which results in the collection of blood leaking from the artery and the formation of pseudoaneurysms. In our patient, preoperative CT examinations of the sphenoid sinus showed partial opacification (Figure 1), and the anterior sphenoid wall was carefully removed with bone punches to avoid cracks of the skull base or the posterior sphenoid wall, which can lead to carotid artery injury. Intraoperative endoscopic examination of the sphenoid sinus showed a nearly intact membrane with little inflammation or swelling; thus, the sphenoid sinus was not surgically manipulated (Figure 3). Because ESS had no complications and produced no marked changes in sphenoid sinus construction, we assume that ESS was not related, at least directly, to the diplopia.

Although the CCA in our patient was not been noticed before ESS, when the level and width of the preoperative CT images had been changed to a soft-tissue window, the aneurysm's contour was detected (Figure 4) and indicated that the aneu- 

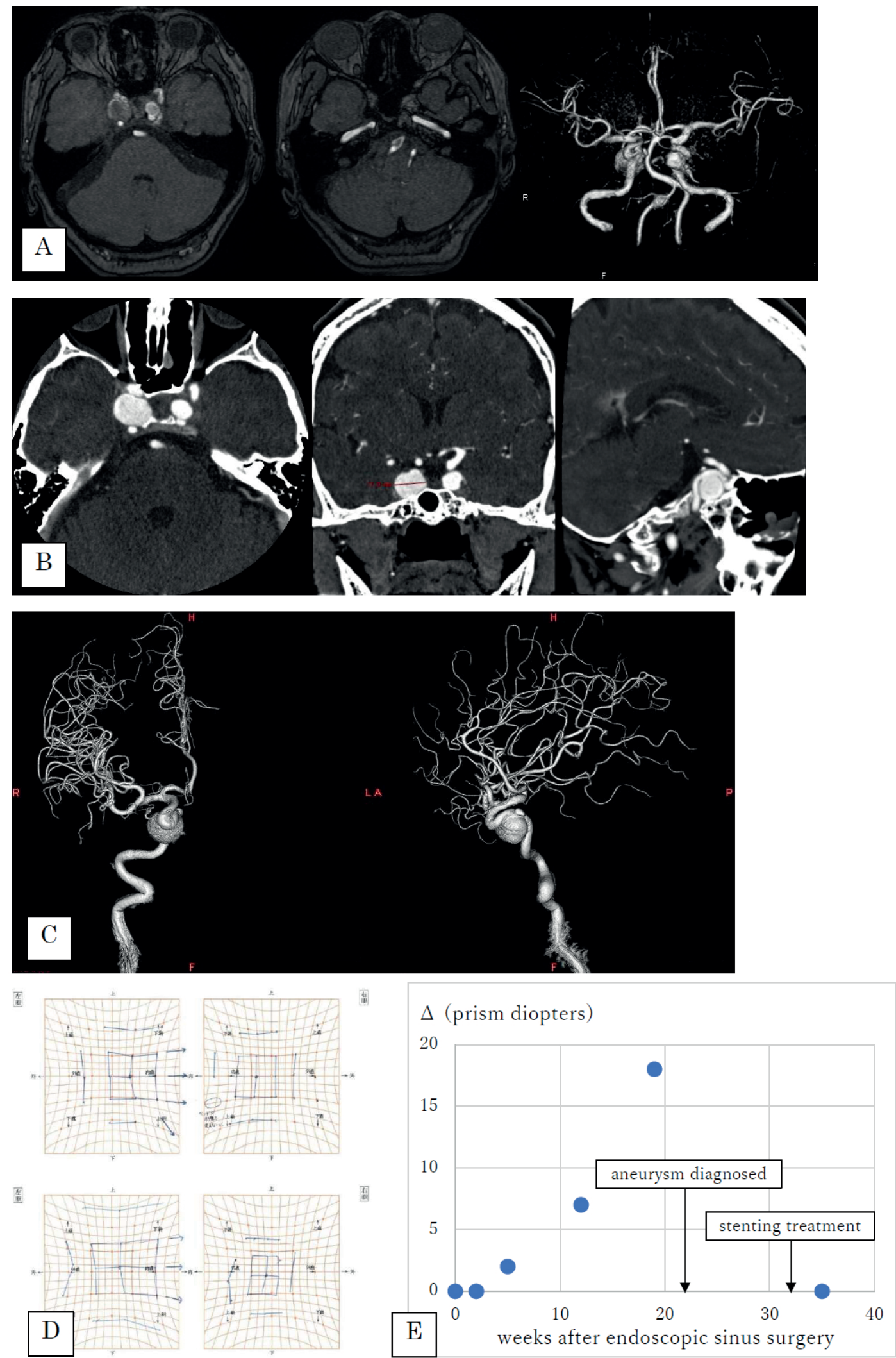

Figure 2. Image findings of cavernous carotid aneurysms and the Hess chart. (A) Magnetic resonance angiography of aneurysms (bilateral cavernous carotid aneurysms [CCAs] and a right vertebral aneurysm; right CCA: $17 \times 13 \mathrm{~mm}$; left CCA: $10 \times 8 \mathrm{~mm}$ ) (B) Computed tomography (CT) with contrastenhancement of CCA (axial, coronal, and sagittal plane of CCA. Maximum diameter $=17 \mathrm{~mm}$.) (C) Digital subtraction angiography with a CT angiogram of the right internal carotid artery clearly shows an aneurysm of the cavernous sinus and its origin. (D) Hess charts obtained before (left) and after (right) stenting treatment. Diplopia decreased from $12^{\Delta}$ to $4^{\Delta}$. 

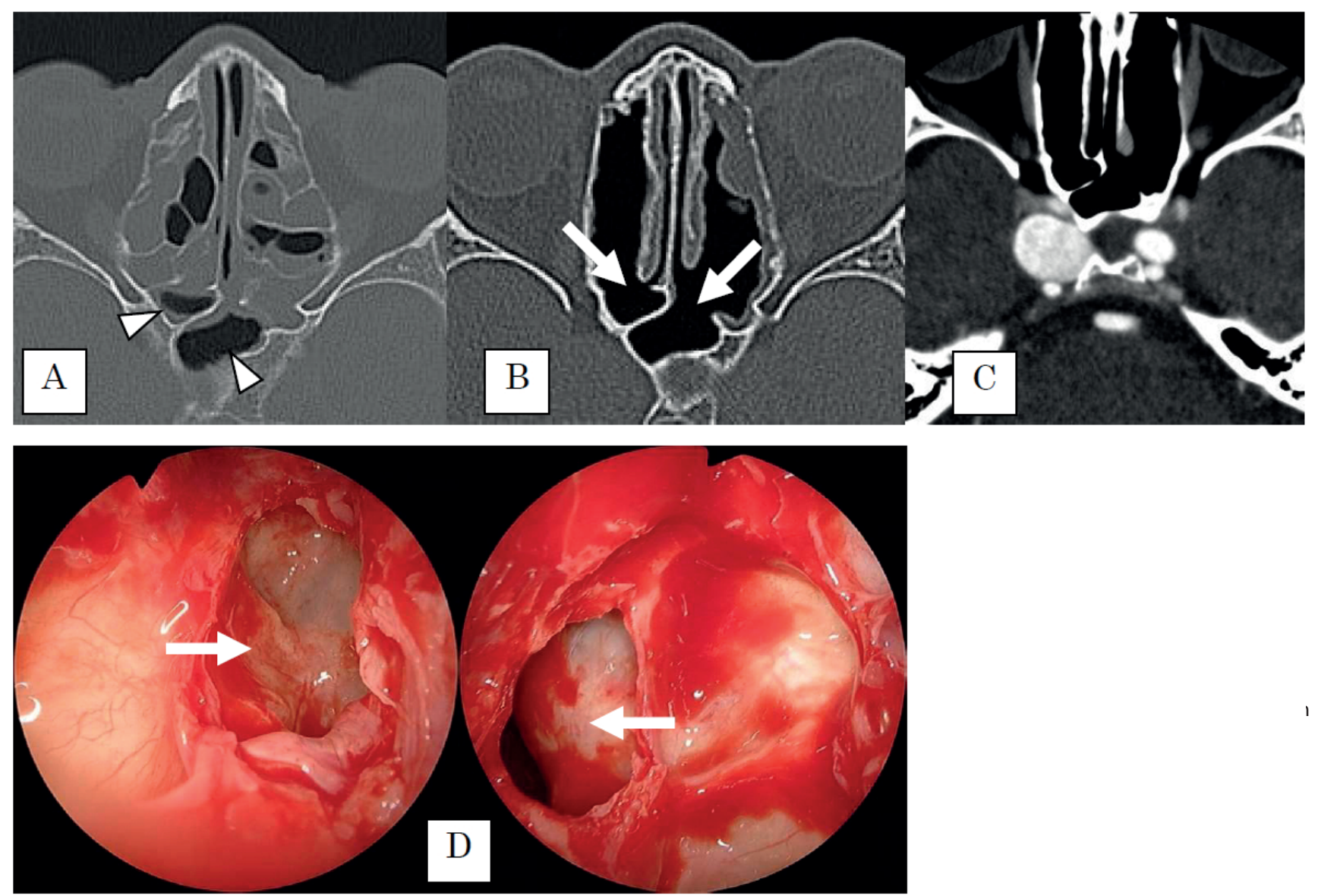

Figure 3. Preoperative and postoperative computed tomography and intraoperative endoscopic findings of the sphenoid sinus. (A) Preoperative computed tomography (CT) of the sphenoid sinus. A sphenoethmoid (Onodi) air cell is seen on the left side. Arrowheads show partial opacifications in bilateral sphenoid sinuses. (B) Postoperative CT of the sphenoid sinus. Arrows show removal of the anterior sphenoid wall, otherwise no bony destruction of the sphenoid sinus. (C) Postoperative CT with contrast enhancement shows neither bone thinning nor protrusion of the cavernous carotid aneurysm to the sphenoid sinus. (D) Intraoperative endoscopic findings of the sphenoid sinus. Arrows show bilateral sphenoid sinus with little sign of mucous edema.

rysm had developed before ESS. However, we did not detect the aneurysm before ESS; if we had, we would have consulted a neurosurgeon. Furthermore, CCA is usually diagnosed with a $\mathrm{CT}$ angiographic examination, with some studies using magnetic resonance imaging without contrast enhancement (8) but no studies having supported the use of $\mathrm{CT}$ without contrast enhancement. The contour of the aneurysm in our patient was detected after ESS, but noticing the aneurysm before ESS was difficult owing to the aneurysm's low contrast.

The natural history of CCA is usually benign ${ }^{(9)}$. The rate of rupture is lower than that of other intracranial aneurysms. Should a rupture occur, it generally forms a carotid artery-cavernous sinus fistula, rarely developing to subarachnoid hemorrhage but occasionally causing secondary epistaxis ${ }^{(10,11)}$. The survival rate of CCA is high, and the main symptom is cranial nerve paralysis via a mass effect. Therefore, CCA is often followed up with imaging studies and treated when cranial nerve symptoms develop. A recent study has found that patients with CCA are more likely to be female and to have a lower incidence of hypertension than do patients with intracranial berry aneurysms and that the risk of growth is associated with aneurysm size ${ }^{(12)}$. According to this study, the aneurysm in our patient, which had a diameter of $17 \mathrm{~mm}$, would be classified as large/giant with a growth risk of $19.2 \%$ per patient-year. Therefore, the aneurysm in our patient had a high risk of enlarging and causing cranial nerve symptoms.

Whether the ESS performed in our patient affected aneurysm growth and led to diplopia remains unclear. Hypertension can be a risk factor for aneurysms, but during the surgery the patient's blood pressure was well maintained and even had to be elevated several times with ephedrine. However, the patient's mean blood pressure during the 6-day hospital stay of 139/84 $\mathrm{mm} \mathrm{Hg}$ might suggest previously unnoticed hypertension. Another possible cause of aneurysms is bacterial infection. For example, an internal carotid aneurysm developing after sinusitis and resulting in multiple cranial nerve palsy has been reported 


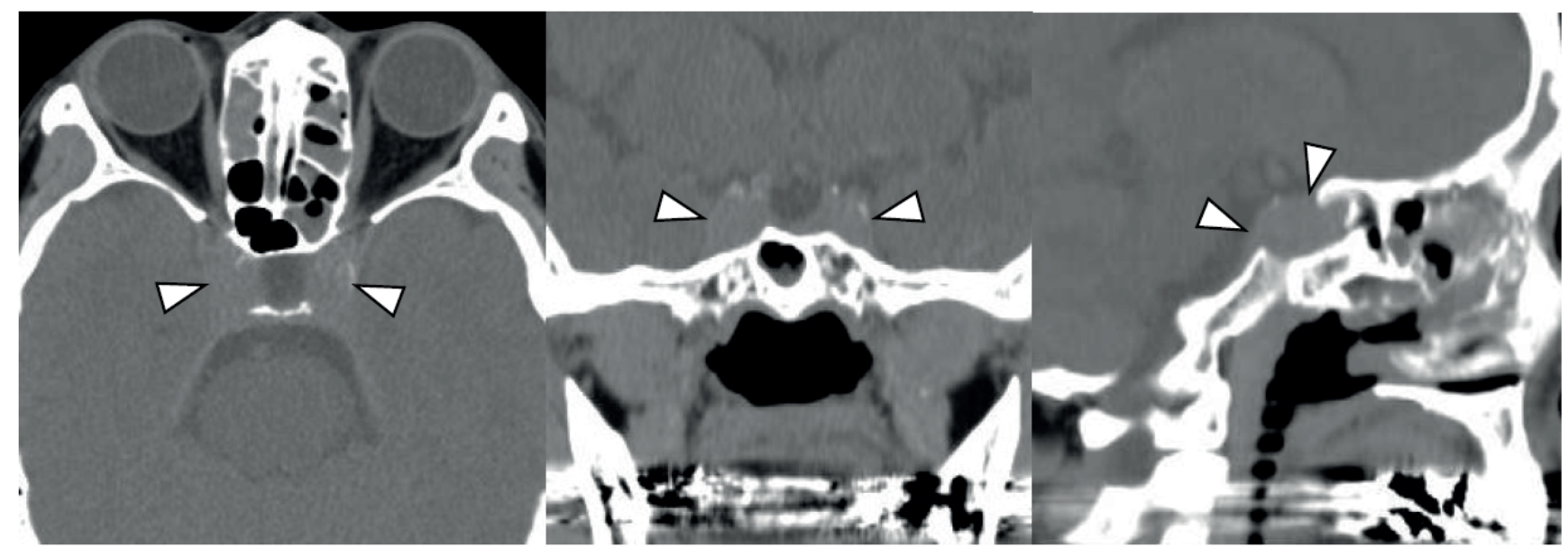

Figure 4. Preoperative computed tomography without contrast enhancement of the cavernous sinus. Arrowheads show the contour of the cavernous carotid aneurysm. The aneurysm is detectable in a soft tissue window of the coronal and sagittal planes but is difficult to detect in the axial plane.

(13). Many cases of sinusitis-related aneurysm have been reported, but the condition most often related is sphenoid sinusitis. Because the $\mathrm{CT}$ and endoscopic examinations in our patient showed no signs of severe sphenoid sinusitis, the likelihood of sphenoid sinusitis-related aneurysm is decreased. Ampicillin was administered as a prophylactic antibiotic, and there were no signs of surgical site infection. Nevertheless, sinusitis was present in the sinuses, and because the surgery itself increased the risk of bacterial infection, which can cause an aneurysm, surgery might have increased the growth of the aneurysm.

\section{Conclusions}

We have reported a case of intracranial aneurysm diagnosed when diplopia developed 2 weeks after ESS had been performed. Possible factors in the growth of the aneurysm were an indirect effect of surgery, perioperative or previously unnoticed hypertension, and bacterial sinusitis. Intracranial aneurysms are asymptomatic unless they cause a mass effect or rupture and are difficult to detect with non-contrast-enhanced CT. A thorough examination, including intracranial imaging, should be performed if diplopia develops after ESS.

\section{List of abbreviations}

CCA: cavernous carotid aneurysm; ESS: endoscopic sinus surgery; CT: computed tomography; ${ }^{\Delta}$ : prism dioptres

\section{Acknowledgments}

Not applicable.

\section{Authorship contribution}

MT wrote the manuscript. EM revised the manuscript. All authors read and approved the final manuscript.

\section{Conflict of interest}

The authors declare that they have no competing interests.

\section{Funding}

Not applicable.

\section{Consent for publication}

Written informed consent for publication of clinical details and clinical images was obtained from the patient.

\section{Availability of data and materials}

Not applicable.

\section{References}

1. May M, Levine HL, Mester SJ, Schaitkin B. Complications of endoscopic sinus surgery: Analysis of 2108 patients--incidence and prevention. Laryngoscope. 1994;104(9):1080-1083.

2. Ramakrishnan VR, Kingdom TT, Nayak JV, Hwang PH, Orlandi RR. Nationwide incidence of major complications in endoscopic sinus surgery. Int Forum Allergy Rhinol. 2012;2(1):34-39.

3. Suzuki S, Yasunaga $H$, Matsui H, Fushimi
K, Kondo K, Yamasoba T. Complication rates after functional endoscopic sinus surgery: Analysis of 50,734 japanese patients. Laryngoscope. 2015;125(8):1785-1791.

4. Dubey A, Sung WS, Chen YY, Amato D, Mujic A, Waites P, et al. Traumatic intracranial aneurysm: A brief review. J Clin Neurosci. 2008;15(6):609-612.

5. Wewel J, Mangubat EZ, Munoz L. latrogenic traumatic intracranial aneurysm after endoscopic sinus surgery. J Clin Neurosci. 2014;21(12):2072-2076.
6. Tamura T, Rex DE, Puri AS, Wakhloo AK Surgical iatrogenic internal carotid artery injury treated with pipeline embolization device: Case report and review of the literature. JNET. 2017;11(12):640-646.

7. Ghorbani M, Hejazian E, Nikmanzar S, Chavoshi-Nejad M. Traumatic iatrogenic dissecting anterior cerebral artery aneurysms: Conservative management as a therapeutic option. Br J Neurosurg. 2020:1-3.

8. Yanamadala $V$, Sheth $S A$, Walcott BP Buchbinder BR, Buckley D, Ogilvy CS. 
Non-contrast 3d time-of-flight magnetic resonance angiography for visualization of intracranial aneurysms in patients with absolute contraindications to ct or mri contrast. J Clin Neurosci. 2013;20(8):1122-1126.

9. Wiebers DO. Unruptured intracranial aneurysms: Natural history, clinical outcome, and risks of surgical and endovascular treatment. The Lancet. 2003;362(9378):103-110.

10. Moro $Y$, Kojima $H$, Yashiro T, Moriyama $H$ A case of internal carotid artery aneurysm diagnosed on basis of massive nosebleed. Auris Nasus Larynx. 2003;30(1):97-102.

11. Celil G, Engin D, Orhan G, Barbaros ÇL, Hakan K, Adil E. Intractable epistaxis related to cavernous carotid artery pseudoaneurysm: Treatment of a case with covered stent. Auris Nasus Larynx. 2004;31(3):275278.

12. Vercelli G, Sorenson TJ, Aljobeh AZ, Vine $R$, Lanzino G. Cavernous sinus aneurysms: Risk of growth over time and risk factors. J Neurosurg. 2019;132(1):22-26.

13. Suzuki N, Suzuki M, Araki S, Sato H. A case of multiple cranial nerve palsy due to sphenoid sinusitis complicated by cerebral aneurysm. Auris Nasus Larynx. 2005;32(4):415-419.

\section{Eri Mori}

The Jikei University School of Medi-

cine

ENT / Head and Neck Surgery

3-25-8, Nishi-Shimbashi

Minato-ku

Tokyo 105-8461

Japan

Tel: $+81-(0) 3-3433-1111$

E-mail: sekieri0421@gmail.com

ISSN: 2589-5613 / ( 2020 The Author(s). This work is licensed under a Creative Commons Attribution 4.0 International License. The images or other third party material in this article are included in the article's Creative Commons license, unless indicated otherwise in the credit line; if the material is not included under the Creative Commons license, users will need to obtain permission from the license holder to reproduce the material. To view a copy of this license, visit http://creativecommons.org/ licenses/by/4.0/ 